\title{
Spin Lifetime Enhancement by Shear Strain in Thin Silicon-on-Insulator Films
}

\author{
Dmitry Osintsev, Viktor Sverdlov, and Siegfried Selberherr \\ Institute for Microelectronics, TU Wien, \\ Gußhausstraße 27-29/E-360, 1040 Wien, Austria
}

\begin{abstract}
Spintronics attracts at present much interest because of the potential to build novel spin-based devices which are superior to nowadays charge-based microelectronic elements. Utilizing spin properties of electrons opens great opportunities to reduce device power consumption in future electronic circuits. Silicon, the main element of microelectronics, is promising for spin-driven applications. Understanding the details of the spin propagation in silicon structures is a key for building novel spin-based nanoelectronic devices. We investigate the influence of shear strain on surface roughness induced spin relaxation in a thin silicon-on-insulator-based transistor. Shear strain dramatically influences the spin, which opens a new opportunity to boost spin lifetime in a silicon spin field-effect transistor.
\end{abstract}

\section{Introduction}

With semiconductor device scaling apparently approaching its fundamental limits, new engineering solutions and innovations are required to further improve the performance of microelectronic components. Spin as an additional degree of freedom is promising for future nanoelectronic devices.

Silicon is the primary material for microelectronics. A long spin life time in silicon is a consequence of the weak spin-orbit interaction and the spatial inversion symmetry of the lattice $(1,2)$. In addition, silicon is composed of nuclei with predominantly zero magnetic moment. A long spin transfer distance of conduction electrons has already been demonstrated experimentally (3). Spin propagation at such distances combined with a possibility of injecting spin in silicon at room (4) or even elevated (5) temperatures makes the fabrication of spin-based switching devices quite plausible in the upcoming future. However, the relatively large spin relaxation experimentally observed in electrically-gated lateral-channel silicon structures (1) might become an obstacle in realizing spin driven devices (2). Spin relaxation in silicon thin films has not been addressed yet. Because of the paramount importance of SOI and FinFET 3D technology for technology nodes below $22 \mathrm{~nm}$, understanding details of spin propagation in silicon thin films is urgently needed (6).

In bulk silicon the conduction electrons are positioned close to the minima of the three pairs of valleys near the edges of the Brillouin zone along the X-, Y-, and Z-axes. Each state is described by the valley index, the wave vector $\mathbf{k}$ relative to the valley minimum, and the spin orientation (spin-up $\uparrow$ and spin-down $\downarrow$ ) on a chosen axis. The main mechanism of spin relaxation in bulk silicon is due to the electron-phonon transitions between the spin-up and down states located in the different valley pairs: the f-processes $(6,7)$. Uniaxial [001] stress removes the degeneracy between the $\mathrm{X}(\mathrm{Y})$ - and Z-valleys, and it is thus predicted to boost spin life time in bulk silicon (7). 
In (001) thin silicon films the confinement leads to the formation of an unprimed subband ladder from the Z-valleys and a prime ladder from the $\mathrm{X}(\mathrm{Y})$-valleys. Due to the difference in the quantization energies the f-processes in the unprimed subbands are suppressed even in unstrained films. Thus, spin relaxation due to the intra- and intersubband transitions between the states with opposite spin projections within the unprimed ladder must be considered.

The low field electron mobility in thin films at high carrier concentrations is limited by surface roughness scattering (8). We investigated the behavior of the spin lifetime in strained silicon films taking into account the surface roughness as the major mechanism responsible for spin relaxation. To do so we need to know the subband wave functions and subband energies in the unprimed ladder. A perturbative $\mathbf{k} \cdot \mathbf{p}$ approach $(2,9,10)$ is suitable to describe the electron subband structure in the presence of strain. We utilize a spindependent $\mathbf{k} \cdot \mathbf{p}$ Hamiltonian (9-11). Our effective 4x4 Hamiltonian considers only the relevant [001] oriented valleys with the spin included, which produce the low-energy unprimed subband ladder. Within this model the unprimed subbands in the unstrained (001) film are degenerate without spin-orbit effects included. An accurate inclusion of the spinorbit interaction results in a large mixing between the spin-up and spin-down states in spin "hot spots" along the [100] and [010] axes characterized by strong spin relaxation. These hot spots should be contrasted with the spin hot spots appearing in the bulk system $(2,12)$ : their origin lies in the unprimed subband degeneracy in a confined electron system which effectively projects the bulk spin hot spots located at the edge of the Brillouin zone to the center of the 2D Brillouin zone.

Shear strain lifts the degeneracy between the unprimed subbands (10). The energy splitting between the otherwise equivalent subbands removes the origin of the spin hot spots in a confined silicon system, which should substantially improve the spin lifetime in gated silicon systems.

\section{Model}

We solve numerically the Hamiltonian

$$
H=\left[\begin{array}{ll}
H_{1} & H_{3} \\
H_{3}^{\dagger} & H_{2}
\end{array}\right]
$$

with $H_{1}, H_{2}$, and $H_{3}$ defined as

$$
\begin{aligned}
& H_{j}=\left[\frac{\hbar^{2} k_{z}^{2}}{2 m_{l}}+\frac{(-1)^{j} \hbar^{2} k_{0} k_{z}}{m_{l}}+\frac{\hbar^{2}\left(k_{x}^{2}+k_{y}^{2}\right)}{2 m_{t}}+U(z)\right] I, \\
& H_{3}=\left[\begin{array}{cc}
D \varepsilon_{x y}-\frac{\hbar^{2} k_{x} k_{y}}{M} & \left(k_{y}-k_{x} i\right) \Delta_{S O} \\
\left(-k_{y}-k_{x} i\right) \Delta_{S O} & D \varepsilon_{x y}-\frac{\hbar^{2} k_{x} k_{y}}{M}
\end{array}\right] .
\end{aligned}
$$

Here $j=1,2$, $\mathrm{I}$ is the identity $2 \mathrm{x} 2$ matrix, $\mathrm{m}_{\mathrm{t}}$ and $\mathrm{m}_{1}$ are the transversal and the longitudinal silicon effective masses, $M^{-1} \approx m_{t}^{-1}-m_{0}^{-1}, k_{0}=0.15 \times 2 \pi / a$ is the position of the valley minimum relative to the $\mathrm{X}$-point in unstrained silicon, $\varepsilon_{x y}$ denotes the shear strain component, and $\mathrm{D}=14 \mathrm{eV}$ is the shear strain deformation potential. 


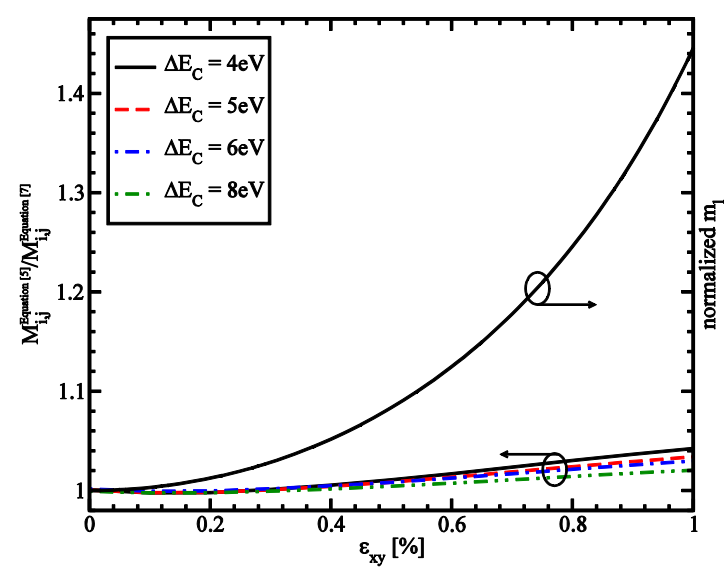

Figure 1. Dependence of the ratio of the surface scattering elements calculated by [5] to the surface scattering elements calculated by [7] and longitudinal effective mass normalized to $m_{l}$ at zero strain value for the film thickness $2.48 \mathrm{~nm}$, for $k_{x}=0.25 \mathrm{~nm}^{-1}$, and $k_{y}=0.25 \mathrm{~nm}^{-1}$.

The spin-orbit term $\tau_{y} \otimes\left(k_{x} \sigma_{x}-k_{y} \sigma_{y}\right)$ with

$$
\Delta_{S o}=\frac{\hbar^{2}}{2 m_{0}^{3} c^{2}}\left|\sum_{n} \frac{\left\langle X_{1}\left|p_{j}\right| n\right\rangle\left\langle n\left|[\nabla V \times p]_{j}\right| X_{2 \prime}\right\rangle}{E_{n}-E_{X}}\right|,
$$

couples states with the opposite spin projections from the opposite valleys. $\sigma_{x}$ and $\sigma_{y}$ are the spin Pauli matrices and $\tau_{y}$ is the $y$-Pauli matrix in the valley degree of freedom and $\mathrm{c}$ is the speed of light. In the Hamiltonian [1] $U(z)$ is the confinement potential, and the value $\Delta_{S O}=1.27 \mathrm{meVnm}$ computed by the empirical pseudopotential method (11) is close to the one reported by Li and Dery (2).

In the case of non-degenerate parabolic band dispersion and the infinite barrier at the silicon-oxide interface the surface roughness scattering matrix elements are proportional to the product of the subband function derivatives at the interface (15)

$$
M_{i, j}(\boldsymbol{q})=\left[\frac{\hbar^{2}}{2 m_{l}} \frac{\mathrm{d} \xi_{i}(z)}{\mathrm{dz}} \frac{\mathrm{d} \xi_{j}(z)}{\mathrm{dz}}\right]_{z= \pm \frac{t}{2}} \Delta(\boldsymbol{q})
$$

Here $\Delta(\boldsymbol{q})$ is the Fourier transform of the random variation of the interface position with respect to the in-plane $\mathbf{r}$ coordinate, $\mathbf{q}$ is the wavevector change due to scattering, $\xi_{i, j}$ are the subband eigenfunctions in a silicon film, and $t$ is the film thickness. The generalization of this expression to the $\mathbf{k} \cdot \mathbf{p}$ theory when the subband functions consist of several components is required. An additional difficulty appears in strained films where the longitudinal mass is the function of shear strain

$$
m_{l}\left(\varepsilon_{x y}\right)=\frac{m_{l}\left(\varepsilon_{x y}=0\right)}{1-\left(\frac{D \varepsilon_{x y}}{\frac{\hbar^{2} k_{0}^{2}}{m_{l}\left(\varepsilon_{x y}=0\right)}}\right)^{2}} .
$$

In order to resolve the question which mass to use, we follow an alternative approach by Esseni (14), where the surface roughness intrasubband scattering matrix elements are

$$
M_{i, i}(\boldsymbol{q})=\left[\Delta E_{C} \xi_{i}(z) \xi_{j}(z)\right]_{z= \pm \frac{t}{2}} \Delta(\boldsymbol{q})
$$




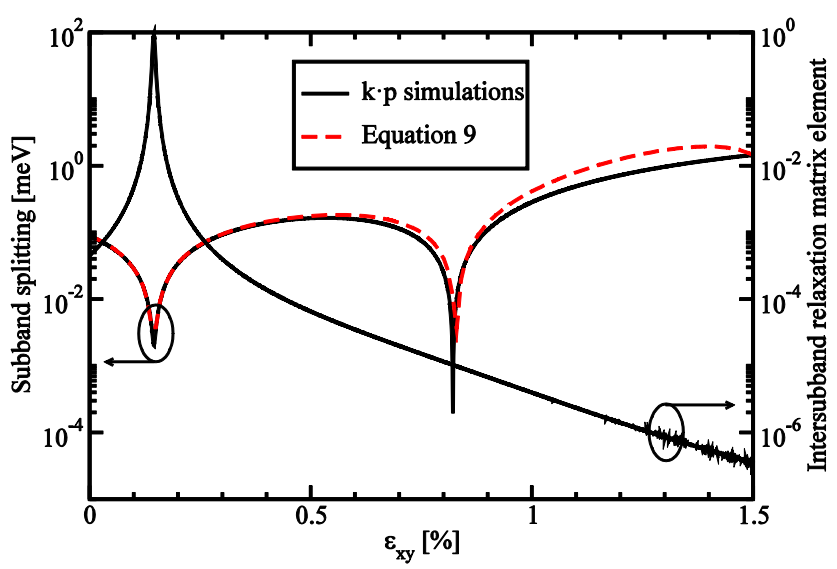

Figure 2. Subband splitting and intersubband relaxation matix elements for the film thickness $4 \mathrm{~nm}$, the conduction band offset is $4 \mathrm{eV}, k_{x}=0.5 \mathrm{~nm}^{-1}, k_{y}=0.1 \mathrm{~nm}$

In the limit of $\Delta E_{C} \rightarrow \infty$ equations [5] and [7] must produce the same result $(14,15)$.

Figure 1 shows the dependence of the longitudinal effective mass obtained from the ratio of the surface scattering elements calculated with [5] and [7], for several values of $\Delta E_{C}$, as a function of shear strain. Also the dependence of the longitudinal effective mass normalized to the $m_{l}$ at zero strain on the shear strain according to [6] is shown.

We observe that the effective mass obtained from the ratio of the matrix elements calculated with two different methods changes only a little with strain. These changes are becoming smaller for the conduction band offset increased. It means that the longitudinal effective mass in [5] must not depend on shear strain. Therefore, within the $\mathbf{k} \cdot \mathbf{p}$ method the surface roughness scattering matrix elements are generalized as

$$
M_{i, j}(\boldsymbol{q})=\left[\frac{\hbar^{2}}{2 m_{l}\left(\varepsilon_{x y}=0\right)}\left(\frac{\mathrm{d} \xi_{i}(z)}{\mathrm{dz}}, \frac{\mathrm{d} \xi_{j}(z)}{\mathrm{dz}}\right)\right]_{z= \pm \frac{t}{2}} \Delta(\boldsymbol{q})
$$

where $(a, b)$ is the scalar product between the two multi-component subband functions $a$ and $b$. An expression similar to [8] has been employed in the six-band k·p calculations (16).

By a unitary transformation the Hamiltonian [1] can be transformed into canonical form with $H_{j}=\left[\frac{\hbar^{2} k_{z}^{2}}{2 m_{l}}+\frac{\hbar^{2}\left(k_{x}^{2}+k_{y}^{2}\right)}{2 m_{t}}+(-1)^{j} \delta\right] I$ and $H_{3}=\left[\frac{\hbar^{2} k_{0} k_{z}}{m_{l}}\right] I$, were $\delta=\sqrt{\left(D \varepsilon_{x y}-\frac{\hbar^{2} k_{x} k_{y}}{M}\right)^{2}+\Delta_{S o}^{2}\left(k_{x}^{2}+k_{y}^{2}\right)}$. Following (10), we find the energy dispersion of the lowest conduction bands of a square well potential with infinite walls as

$$
\Delta E_{n}=\frac{2 y_{n}^{2} \delta}{k_{0} t \sqrt{\left(1-y_{n}^{2}-\eta^{2}\right)\left(1-y_{n}^{2}\right)}}\left|\sin \left(\sqrt{\frac{1-y_{n}^{2}-\eta^{2}}{1-y_{n}^{2}}} k_{0} t\right)\right| .
$$

Here $y_{n}=\frac{\pi n}{k_{0} t}$ and $\eta=\frac{m_{l} \delta}{\hbar^{2} k_{0}^{2}}$. This expression generalizes the corresponding dispersion relation (13) by including shear strain and spin-orbit interaction. 


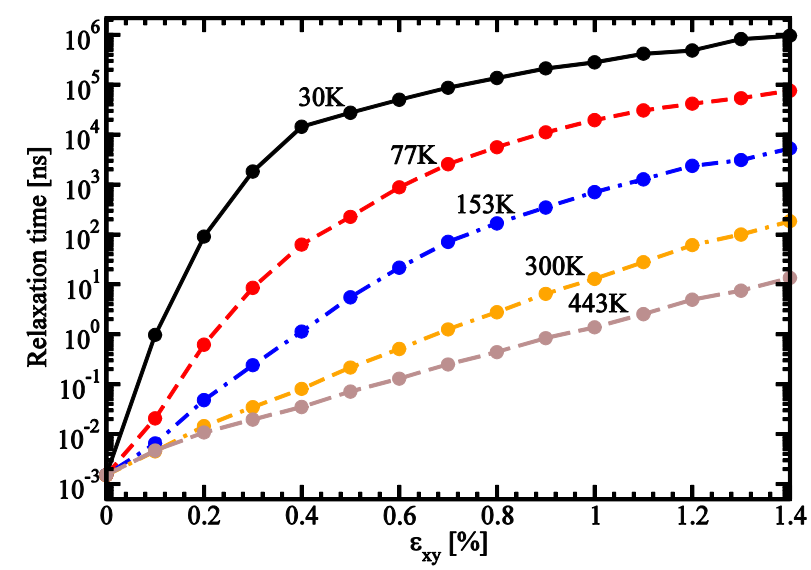

Figure 3. Spin lifetime for a film thickness of $2.48 \mathrm{~nm}$ and an electron density of states $10^{12} \mathrm{~cm}^{-2}$ for different temperatures.

Figure 2 shows the splitting between the equivalent unprimed subbands (the valley splitting) obtained numerically with the $\mathbf{k} \cdot \mathbf{p}$ approach in comparison to the analytical Equation [9]. The results are in good agreement. As follows from Equation [9] the splitting between the subbands depends on $D \varepsilon_{x y}-\frac{\hbar^{2} k_{x} k_{y}}{M}$, and the degeneracy between the unprimed subbands is increased, when this term is nonzero. For $k_{x}=0.5 \mathrm{~nm}^{-1}, k_{y}=0.1 \mathrm{~nm}^{-1}$ the strain value $0.116 \%$ causes this term to vanish and minimizes the valley splitting, in good agreement with the first sharp valley splitting reduction in Figure 2. The valley splitting is also proportional to $\left|\sin \left(\sqrt{\frac{1-y_{n}^{2}-\eta^{2}}{1-y_{n}^{2}}} k_{0} t\right)\right|$. The second minimum in the valley splitting in Figure 2 is because of the zero value of the $\left|\sin \left(\sqrt{\frac{1-y_{n}^{2}-\eta^{2}}{1-y_{n}^{2}}} k_{0} t\right)\right|$ term.

The dependence of the intersubband spin relaxation matrix elements [5] on shear strain is also shown in Figure 2. The spin relaxation matrix elements increase until the strain value $0.116 \%$, the point determined by the spin hot spot condition. Applying strain larger than $0.116 \%$ suppresses spin relaxation significantly. The relaxation matrix elements demonstrate a sharp feature only for the shear strain value of $0.116 \%$.

Spin relaxation rates are calculated in the following way $(2,16)$

$$
\begin{gathered}
\frac{1}{\tau}=\frac{\int \frac{1}{\tau\left(\overrightarrow{k_{1}}\right)} f(\varepsilon)(1-f(\varepsilon)) d \overrightarrow{k_{1}}}{\int f(\varepsilon) d \overrightarrow{k_{1}}}, \\
f(\varepsilon)=\frac{1}{1+e^{\left(\frac{\varepsilon-M}{k T}\right)},} \\
\int d \overrightarrow{k_{1}}=\int_{0}^{2 \pi} \int_{0}^{\infty} \frac{\left|\overrightarrow{k_{1}}(\varphi, \varepsilon)\right|}{\mid \frac{\partial \varepsilon\left(\overrightarrow{k_{1}}\right)}{\partial \overrightarrow{k_{1}} \mid}} d \varphi d \varepsilon, \\
\frac{1}{\tau\left(\overrightarrow{k_{1}}\right)}=\frac{4 \pi}{\hbar} \sum_{i, j=1,2} \int_{0}^{2 \pi} \Delta^{2} L^{2} \frac{1}{\epsilon_{i j}^{2}\left(\overrightarrow{k_{l}}-\overrightarrow{k_{1}}\right)} \frac{\hbar^{4}}{4 m_{l}^{2}}\left[\frac{d \Psi_{\mathrm{ik}_{1}}^{*}}{d z} \frac{d \Psi_{\mathrm{ik}}}{d z}\right]_{z= \pm \frac{t}{2}}^{2} \times
\end{gathered}
$$




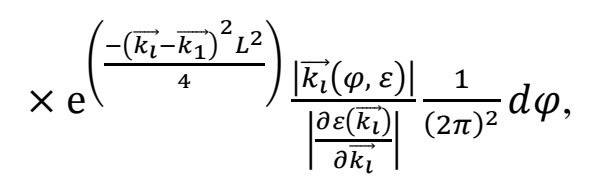

where, $\varepsilon$ is the electron energy, $\overrightarrow{k_{1}}$ is the wave vector, $k$ is the Boltzmann constant, $T$ is the temperature, $M$ is the Fermi energy, $\epsilon_{i j}$ is the dielectric permittivity, $L$ is the autocorrelation length, $\Delta$ is the mean square value of surface roughness fluctuations.

A strong increase of the spin lifetime with shear strain is demonstrated in Figure 3. This increase is a consequence of the fact that shear strain pushes out the regions of large mixing between the spin-up and spin-down states to higher kinetic energies outside of the occupied states.

\section{Conclusion}

We have investigated the lowest unprimed electron subband splitting in a thin SOI film as a function of shear strain and we have demonstrated that the valley splitting minima due to zero values of the sin-like term does not cause a sharp peak of the intersubband relaxation matrix elements, but the minimum due to a vanishing $\boldsymbol{D} \boldsymbol{\varepsilon}_{\boldsymbol{x} \boldsymbol{y}}-\frac{\hbar^{2} \boldsymbol{k}_{\boldsymbol{x}} \boldsymbol{k}_{\boldsymbol{y}}}{\boldsymbol{M}}$ leads to the large mixing between the spin-up and spin-down states. Due to the inter-subband splitting increase, the matrix elements for spin relaxation decrease rapidly with shear strain. Thus, shear strain used to enhance electron mobility can also be used to boost the surface roughness limited spin lifetime by several orders of magnitude.

\section{Acknowledgments}

Our work is supported by the European Research Council through the grant \#247056 MOSILSPIN.

\section{References}

1. J. Li, I. Appelbaum, Phys. Rev. B, 84, 165318 (2011).

2. P. Li, H. Dery, Phys. Rev. Lett., 107, 107203 (2011).

3. B. Huang, D.J. Monsma, I. Appelbaum, Phys. Rev. Lett., 99, 177209 (2007).

4. S.P. Dash et al., Nature, 462, 491-494 (2009).

5. C.H. Li et al., Nat. Comm., 2, 245 (2011).

6. Y. Song, H. Dery, Phys. Rev. B, 86, 085201 (2012).

7. J.-M. Tang, B. Collins, M. Flatte, Phys. Rev. B, 85, 045202 (2012).

8. K. Uchida et al., Intl. Electron Devices Meeting (2002).

9. G.L. Bir, G.E. Pikus, Symmetry and strain-induced effects in semiconductors. New York/Toronto: J. Wiley \& Sons 1974.

10. V. Sverdlov, Strain-induced effects in advanced MOSFETs. Wien - New York. Springer 2011.

11. D. Osintsev et al., Intl. Workshop on Computational Electronics (2012).

12. J.L. Cheng, M.W. Wu, J. Fabian, Phys. Rev. Lett., 104, 016601 (2010).

13. V. Sverdlov et al., J. Comput. Electronics, 8, 3-4 (2009).

14. D. Esseni, Trans. On Electron Devices, 51(3), 394-401(2004).

15. S. Jin, M.V. Fischetti, T.-W. Tang, Trans. On Electron Devices, 54(9), 2191-2203 (2007).

16. M. V. Fischetti et al., J. Appl. Phys., 94, 1079 (2003). 